\title{
Study of total serum lactate dehydrogenase activity as an indirect evidence of acute Plasmodium falciparum infection
}

\author{
Dr Surendra Khosya*,Dr Ramraj Meena*, Dr Hariom Meena* \\ $3^{\text {rd }}$ year Resident in Internal Medicine.RUHS( Rajasthan university of health sciences) \\ Department of medicine. MBS and associated hospitals, Government Medical College, Kota, Rajasthan,334001.
}

\begin{abstract}
Background : Lactate dehydrogenase ( $L D H)$ activity was assayed in the sera of 40 adult male and 40 adult female patients within the age group of 16-50 years presenting with acute, Plasmodium falciparum malaria infection and a control group of 40 healthy adults within the same age group.

Study objective: Study was design to determine diagnostic value of serum LDH activity in patient with acute P.falciparum Infection.

Methods: Patient selection and pre-qualification were done by simple random sampling of individuals presenting at the MBS Hospital Outpatient Department with a history of fever and malaise within a period of one to eight days, and who were confirmed to be infected with the P. falciparum malaria parasite by microscopically examination of Giemsa-stained thin blood slides.

Results: The mean serum LDH activity in male patients was found to be $564 \pm 236.0$ IU. This activity is significantly higher than the control LDH activity of 237.10 $\pm 19.0 \mathrm{IU}$ (p-value is less than 0.05). The mean serum $\mathrm{LDH}$ activity among female patients was $468 \pm 177.0 \mathrm{IU}$, which is a relatively higher activity compared to the control LDH activity of $237.10 \pm 19.0 \mathrm{IU}$ (p-value is less than 0.05).

Conclusion: The combination of acute hepatocellular injury and red cell haemolysis induced by the invading merozoites may account for the increase in serum LDH activity during this infection. Therefore serum LDH activity is a potentially valuable enzymatic marker of falciparum malaria infection.
\end{abstract}

\section{Introduction}

Lactate dehydrogenase (LDH) is an intracellular enzyme, which catalyses the readily reversible reaction involving the oxidation of lactate to pyruvate with nicotinamide adenine dinucleotide (NAD) serving as coenzyme $^{1}$. LDH is an enzyme, which is classified as a true intracellular Enzyme ${ }^{2}$ because of its high degree of tissue specificity where overall tissue concentrations are some 500-fold greater than serum levels under normal circumstances ${ }^{3}$. LDH have five theoretically possible forms, which are found in human tissues e.g. liver, heart, erythrocytes, skeletal muscles and kidneys ${ }^{4}$. So disease affecting these organs such as renal infarction myocardial infarction and haemolysis have been reported to be associated with significant elevations in total serum LDH activity. Such elevations have been widely applied as diagnostic indices for kidney, liver, heart and red blood cell dysfunction ${ }^{5-7}$. Additionally, high serum LDH activity has also been reported in small cell carcinoma of the lung, nephroblastoma, neuroblastoma and metabolic neuroendocrine tumour ${ }^{8}$ measles

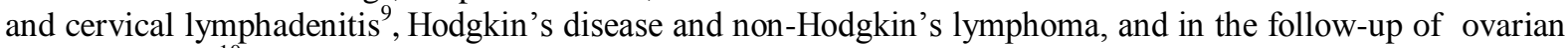
Dysgerminoma $^{10}$.

Plasmodium falciparum malaria infection is a febrile illness accounting for 300-500 million clinical cases annually worldwide. The life cycle of this parasite in the human host includes the developmental cycle in red blood cells, and the cycle taking place in the liver cell parenchyma, includes a series of transformations in the host hepatocytes. Pathophysiological processes usually associated with acute P. falciparum malaria infections, i.e., the hepatic activity of the invading sporozoites leading to centrilobular liver damage and the destruction of the host red blood cells consequent to erythrocytic merogony ${ }^{11}$. Being rich sources of LDH, the acute liver injury and red blood cell destruction will be followed by the release of LDH into the circulation. This finding has important implications because it highlights the potential of using serum LDH activity as an index in the monitoring of acute P. falciparum malaria infection, particularly when all other possible causes of increased serum LDH levels have been eliminated

\section{Material and method \\ Aims and objective \\ 1. To determine potential of using serum LDH activity as an index in the monitoring of acute P.falciparum infection.}

2. To determine diagnostic value of serum LDH activity in patient with acute P.falciparum infection. 
Study was conducted at MBS Hospital Kota during month of July to September when malaria endemicity is at its highest peak because of highest average rainfall.

Patient selection and prequalification were done by simple random sampling of individual admitted in medicine wards at MBS Hospital Kota. Total 80 patients included in our study among them 40 were males and 40 were females, 40 person also included as control group.

\section{Exclusion criteria:-}

Patient, whose case history showed a concomitant presentation with the following condition :- Acquired immune deficiency syndrome,

Anemia (chronic anemia due to hypo proliferative, hemolytic and other known hemolytic disorder.)

Liver cirrhosis

Alcoholism

Kidney disorder

Patient on self-medication with any anti-malarial drugs prior to presentation.

Malignancy.

\section{Methods}

Venous blood $(5 \mathrm{ml})$ was obtained from each of the patients by venepuncture of the antecubital vein using a sterile needle and syringe between eight and ten o'clock in the morning. The blood samples were then transferred into clean, sterile centrifuge tubes and allowed to clot.

Enzyme assay was carried out within 24 hours of collection. Statistically method used was unpaired Student $t$ test and variable by using Graph in stat version 3.10. was used to calculate $\mathrm{p}$ values. $\mathrm{P}$ value of $<0.05$ was considered to be statistically significant and value $<0.001$ considered as highly significant.

\section{Observation}

Total 80 hospitalized patient (male 40 and female 40) within the age group of 16-50 years presenting with acute, uncomplicated Plasmodium falciparum malaria infection and a control group of 40 healthy adults within the same age group were included in our study.

Table I. Serum LDH activity in adult male and female $P$. falciparum malaria patients and controls.

\begin{tabular}{|l|l|l|}
\hline S.N. & SUBJECT & MEAN SERUM LDH ACTIVITY (IU) \\
\hline 1. & Male patients $(\mathrm{n}-40)$ & $564 \pm 236.0 \mathrm{IU}$. \\
\hline 2. & Female patients $(\mathrm{n}-40)$ & $468 \pm 177.0 \mathrm{IU}$, \\
\hline 3. & Controls $(\mathrm{n}-40)$ & $237.10 \pm 19.0$ \\
\hline
\end{tabular}

\section{Discussion}

In Our study the mean serum LDH activity in male patients was found to be $564 \pm 236.0$ IU. This was more than two times above the control LDH activity of $237.10 \pm 19.0$ IU. Similarly, in female patients, the serum LDH activity of $468 \pm 177.0$ IU is over twice of the control serum LDH activity. Mean serum LDH level was significantly higher in patient infected with $\mathrm{p}$. falciparum infection than control group $(\mathrm{p}<0.05)$. Among the patients, the males were found to have a significantly higher serum LDH. Our results were similar to the study by Garba et $\mathrm{al}^{12}$ in which mean serum LDH activity in male and female patient were 789.0 and 634.0, respectively. Maegraith ${ }^{11}$ postulated that the factors involved in hepatic dysfunction in acute $P$. falciparum malaria infection involve a synergy between local circulatory failure and centrilobular cellular damage. Since LDH is found in clinically-significant amounts both the liver and red blood cells, the observed increase in serum LDH activity during acute $P$. falciparum malaria infection in this study can be accounted for by a synergy between the two pathophysiological processes usually associated with acute $P$. falciparum malaria infections, i.e., the hepatic activity of the invading sporozoites leading to centrilobular liver damage and the destruction of the host red blood cells consequent to erythrocytic merogony ${ }^{11}$. Being rich sources of LDH, the acute liver injury and red blood cell destruction will be followed by the release of LDH into the circulation. This finding 
has important implications because it highlights the potential of using serum LDH activity as an index in the monitoring of acute $P$. falciparum malaria infection, particularly when all other possible causes of increased serum LDH levels have been eliminated.

Although, diagnosis of malaria rest on the demonstration of asexual forms of the parasite in stained peripheral blood smear. Sometimes no parasites can be found in peripheral blood smears from patients with malaria, even in severe infections. This may be explained by partial antimalarial treatment or by sequestration of parasitized cells in deep vascular beds ${ }^{13}$. Interpretation of blood smear films require some experience because artifacts are common. Before a thick smear is judged to be negative,100-200 fields should be examined under oil immersion. So indirect evidences for diagnosis of malaria becomes only the reasons to start or to justify treatment against malaria ${ }^{14}$.

LDH present abundantly in tissues(liver,red bllod cells) which get infected by malarial parasite during completion of asexual cycle. So raised serum LDH level may be considered as an evidence for P. falciparum infection.

\section{Refrences}

[1] Stryer L. Biochemistry. 2nd ed. New York: WH Freeman, 1982

[2] Sullivan JM, Alpers JP. In vitro regulation of rat heart5'-nucleotidase by adenine nucleotides and magnesium. J Bio Chem 1971; 246:3057-63.

[3] Podlasek SJ, McPherson RA. Streptokinase binds lactatedehydrogenase subunit-M, which shares an epitope with plasminogen.Clin Chem 1989; 35:69-73.

[4] Calbreath DF. Clinical Chemistry. Philadelphia: WB Saunders, 1992.

[5] Wills MR. The Biochemical Consequences of Chronic Renal Failure.New York: Harvey, Miller and Medcalf, 1971.

[6] Timmis AD, Nathan AW. Essentials of Cardiology. Oxford: Blackwell Scientific, 1993.

[7] Castaldo G, Oriani G, Cimino L, et al. Total discrimination of peritoneal malignant ascites from cirrhosis- and hepatocarcinomaassociated ascites by assays of ascitic cholesterol and lactatedehydrogenase. Clin Chem 1994; 40:478-83.

[8] Kanowski D, Clague A. Increased lactate dehydrogenase isoenzyme-1in a case of glucagonoma. Clin Chem 1994; 40:158-9.

[9] Sugaya N, Kanno J, Nirasawa M, et al. Increased activities of cytosolaminopeptidase and lactate dehydrogenase in serum originate from lymphocytes in necrotizing lymphadenitis. Clin Chem 1990; 36:304-6.

[10] Pressley RH, Muntz HG, Falkenberry S, et al. Serum lactic dehydrogenaseas a tumour marker in dysgerminoma. Gynecol Oncol $1992 ; 4: 281-3$.

[11] Maegraith B. Aspects of the pathogenesis of malaria. Southeast AsianTrop Med Pub Health 1981; 12:251-67.

[12] I H Garba, G A UbomTotal serum lactate dehydrogenase activity in acute Plasmodium falciparum malaria infection. Singapore Med J 2005; 46(11) : 632

[13] Noppadon Tangpukdee, Chatnapa Duangdee, Polrat Wilairatana, Srivicha Krudsood. Malaria Diagnosis: A Brief Review Korean J Parasitol. 2009 Jun;47(2):93-102.

[14] availableathttp://www.parasitol.or.kr/kjp/search.php? where=aview\&id=28586\&code=0066KJP\&vmod.

[15] Nicholus J.White,Joel G.bremen. Malaria.Fauci AS, Braunwald E, kasper DL, longo DL, hauser SL,Jameson JL et al editorsHarrison's Principles of Internal Medicine. Vol 1. 17th ed. New York: Mc Graw Hill Inc,2008: 1280-94. 